\title{
The Model of the Evolution of the Knowledge Content and Contemporary Science Education Crisis
}

\author{
Tadeusz Wibig \\ Physics Department, University of Łódź \\ Pomorska 149/153, 90-236 Łódź, Poland. \\ Email: wibig@zpk.u.lodz.pl \\ Punsiri Dam-o \\ Physics Department, University of Łódź \\ Pomorska 149/153, 90-236 Łódź, Poland. \\ Email: punsiri_pla@hotmail.com
}

\begin{abstract}
We try to diagnose the situation in science education in the beginning of the 21st century. The coincidence of the occurrence of the global science education crisis and rapid acceleration of development of the science itself allows us to derive a differential equation describing basic patterns of development of human knowledge, using a few quite obvious parameters defined by social and biological determinants of the given moment in history. We then examine the proposed solutions of the general educational problem. Predictions about the evolution of knowledge/science give us the ability to reject some of them, and the analysis of social needs, seems to lead to the one, which we called a "three-way system"
\end{abstract}

Index Terms-Science Education, knowledge system, knowledge evolution.

\section{INTRODUCTION}

The teaching physics and, more broadly: natural sciences complemented by an ambitious and fashionable addition: "modern technologies" at the beginning of the new millennium, according to the common feeling, requires a significant revitalization. We would like to present here the description of the eventual proposition, a system that could be called a "three-way system". It seems to be the logical consequence of the general and objective analysis of changes concerning the future development of societies, which was launched at the dusk of the previous millennium.

The paper is organized as follows: in the Introduction section the basic concepts of the knowledge system evolution are shortly presented and the socio-historical background of the general, global analysis of the problem is presented. In the next section the detailed model of the knowledge evolution is build. The significance and importance of each term in the final equation is illustrated in details. In the following sections the final equation is discussed and applied to the present situation in the science education in particular. The problems ahead of the education system are the consequence of the social evolutions driven by the science and technology development described by our evolution equation. The proposed solution for the education crisis in the form of 'three-way' system is introduced and it is shown that it is in accord with the contemporary state of the society and its needs.

In particular these trends have led to changes in social demand for science and technology education. It is a kind of a paradox that the development of science never seen before conducted in such a short time scale, of practically one generation, became the cause of the collapse of the education system, which had originally driven this development.

This feedback is the result of a long-lasting process. Maybe we will be able to see its following effects, but also they could appear for the next generations. The answer to the question why this mechanism has become active just now is crucial for the right understanding of the problem and thus, finding the right solution. It is the convolution of actual social conditions triggered the observed for some time crisis of the model of education the model which remains, generally, in the same form for a few hundreds of years. We do not wish to go deep into the knowledge creation process. The ideas of Nonaka . and his co-workers [1-7], who created a consistent body of theory concerning knowledge creation in organizations cannot be directly adopted for the global knowledge of the mankind as a whole, especially when analyzing its temporal variations [8]. As it is mentioned by Harsh in [9] any conversion or transfer of knowledge consumes time, which does not appear as a variable in the Nonaka's knowledge dynamics model. The temporal features of the knowledge creation can be seen using the Nissen model [10], which is the extension of the socializationexternalization-combination-internalization picture (e.g., [11]). However, details observed in the human/organization timescale are irrelevant for the global scale. 
The metaphorical analysis for knowledge dynamics using the main concepts of energy [12], or thermodynamics [13], could give the global solution, but as the second law of thermodynamic such treatment is of statistical nature, and works well around the state of equilibrium and by definition is not a right tool for describing in details the process of change, the knowledge evolution, what is our subject of interest. Other metaphors [14] could help even less.

The space-time conceptual framework for the knowledge as a evolving system the "diffusion" can take place and can be studied within the organization [15], from the organization where it is created to others [16, 17].

The Information-Space or I-Space idea of Boisot [18, 19] allow one to study the knowledge creation more globally, and it can be, in principle, used for studies on scientific knowledge evolution. The original model is, however, build for relatively small and small scale (in time) systems is rather a kind of the "microscopic model". We are interested in observing the general global, trends, thus quite other treatment of the knowledge/science system is required.

We start our considerations from the verifications of some of many ideas of the McLuhan "Gutenberg Galaxy" [20]. It contains, among the other aspects, also the analysis of the effects of the medium of the interpersonal communication to the human condition in general. We do not have to share McLuhan's conclusions concerning the causal relation between the evolution of the methods of communication and the ways of human perception and schemes of the knowledge creation and collection, at least some of them, but we use his division of the history of mankind into epochs related to the communication modes:

- oral tribe culture,

- manuscript culture,

- Gutenberg Galaxy, and

- electronic age.

The introduction of the last epoch is of the special interest for us. In the year of 1962, when the "Gutenberg Galaxy" appeared, it could not, of course, take into account today's Internet and the World Wide Web. McLuhan could analyse only the effects of wide expansion of radio and television, and computers (big 'mainframes') are only mentioned marginally. If he could wait a while the Gutenberg Galaxy would come out quite different. Half a century later we can go further and a bit aside, and analyze the consequences of the global communication network, as we have it today, on the human social conditions with the special interest put on the education purposes, and more specific: science (physics) education.

\section{A. Oral Culture}

The time when the humankind bearing resemblance to the one of our times came into existence, is beyond relevance. The beginning of the oral epoch vanished in the dusk of times which passed, the reason of what is the case of definition rather than reality. So it does not really matter whether the date was $10000 \mathrm{BC}$ or $3000 \mathrm{BC}$. During that epoch, knowledge perceived as sciences these days, undoubtedly clarified itself. Not going into details, we may presume that it was a set of mythical-religious stories, which were interpreted in face of the reality of everyday life based on analogy and similarities which provided the people of those times with answers to the most important questions. The statement that the point and the nature of the most primary questions did not change much since then, seems to be bold, and what actually changed were obviously the answers. However, it seems that the ancient answers might have been far more satisfying for the people of those times, than the answers the science provides us with nowadays.

Knowledge, back then, was stored in minds of chosen individuals. We may call them 'the wise men', and the whole oral epoch may be called also 'the epoch of the wise men'. Those wise men gained knowledge when they were young, from their predecessor, and were supposed to pass it to their successor in an analogous way. That process could lead to distortions, which, however, may be treated as elements of the knowledge development process, enriching it with new contents, providing the wise men were aware of the process. If they were not, then the random fluctuations which lead to depletion of knowledge may be treated as the result of indiscretion during the copying process (passing and remembering the stories). Obviously, cases of vanishing of some lines might occur, meaning a break in the chain of knowledge, however it seems that the system of the oral epoch dealt with such cases by contacts with geographically divided structures. This system have evolved for thousands of years before it finally achieved a stable state, where random vanishing of stories was compensated by slowly appearing new ones. Out of necessity, the range of knowledge in the oral epoch covered only as much as a one, let us say particularly gifted and trained mind can accumulate, which all in all is not much. The development of ideas, thoughts, concepts, if it even was to come into existence, then it could not advance much.

Education of the oral epoch was limited to the process of passing down knowledge from generation to generation. There was no social need of popularization of the knowledge among the society. The answers to all the questions were provided by the always present, on-line as we would say nowadays, wise man. Teaching was a simple and a single-track process. Beautiful is the picture of the past.

\section{B. Manuscript Epoch}

The epoch of manuscripts, which may also be called the epoch of libraries, came into existence after the revolutionary event, meaning when the wise men learnt to write. The invention of writing, is usually dated as fifth century BC, yet there are claims that the first notes were on bones of the upper Pleistocene around 20000 - 30000 $\mathrm{BC}$. Anyway, it is a so distant history, that it is not very relevant nowadays. It does not even matter, how long did it take to introduce the new invention to the knowledge storing system. In some regions of the world, that process 
did not end until recently. What is important, is that in one moment it was not necessary anymore to remember the contents of a story, as it could be encoded permanently (or less permanently, depending on the need) in use of a set of signs. So important for McLuhan, the distinction between pictographic and alphabetical writing, and according to him the resulting basic differences in quality, will be left to the reader without comment for now, yet maybe in case of a more detailed analysis the issue would have to be reconsidered. One way or another, 'the wise men' are extinct and their stories had been written down and created the basic knowledge for the library system. Manuscripts were stored in libraries, were knowledge was sealed off against distortion and destruction far more better than when it was stored in fragile brains of the wise men. The most important feature of the new epoch, however, was that libraries were able to easily receive the newly appearing knowledge. Someone, let us call him 'a scientist', had a chance to make his own input in the system of knowledge. Obviously the system was 'foolproof' and there was no chance of adding something which was not an evaluation of the existing state of knowledge. The scientist was obliged to acquaint himself with the knowledge which was stored in the system and then, and based on that, he broadened the system, most often in form of a comment. As time passed, the amount of information increased and it was necessary both due to the capabilities and resources, to concentrate the libraries in science centres, where scientists who were searching for answers to some relevant questions were able to study, as well as they could add something meaningful to do resources if they wanted to.

Education in the library system required specialisation, meaning that a particular group of people had to be taught how to read and write. The group did not only consist of scholars, but also scribes who copied knowledge for the sake of the people who needed it, among whom there were mainly the wealthy people of this world. For some of them, probably deepening knowledge itself was something more important then possessing a collection of neatly ornamented manuscripts. The knowledge was also needed by the people who did not know how to write or read much, and similarly as it was in the epoch of the wise men, some chosen people with the access to knowledge, provided it to the aforementioned group of people - but this time in a written form. Education of the society was still oral. The greatest need for knowledge was obviously manifested by scholars. Their education was all about letting them near the books and maybe disclosing the contents of those books to them, by the people who read and seen more in their lives. A new parameter for describing the state of resources in the epoch of libraries appears. It is the amount of knowledge gained (or potentially available to be gained) by a scholar. This parameter, as it will prove later, is relevant in terms of a quantitative description of the system.

New ideas infiltrated the new system of education slowly. Whereas in the epoch of the wise men a newly invented story started its life in the moment it was said, the newly written pages had to be read, then copied and distributed among the librarian community, global in a sense, and that process took time. That period covers formation of the idea itself based on stage of getting to know of the existing knowledge, writing down the idea, then reaching the potential readers of the manuscript, including other scientists above all. It is them who finally accept the new idea, or not, and the majority of votes is the factor which defines whether the new idea lands in the set of knowledge of the humankind, or gets forgotten and abandoned somewhere along the way. And that delay, according with the following debate, proves to be a key factor. In the epoch of libraries, the time of distribution of a particular idea may be estimated even as a few hundred years.

\section{Gutenberg Galaxy}

The Gutenberg Galaxy, which from our perspective is the epoch of the printed word, does not qualitatively differ from the previous epoch of manuscripts. In that moment, we face with yet another discrepancy in relation to McLuhan's concept. What in our opinion changes most, is only the previously mentioned delay in proliferation of new ideas. There are only as many copies of a book in a printed form made, as needed. The socio-psychological changes do not have to come from the medium, but can be the result of the environmental changes connected to the easiness and the pace of duplication of ideas. For instance, the position of the author himself, who is a modern type scientist. Coming into existence of new ideas is still based on the primary process of getting acquainted with the existing knowledge. It is increasingly being documented as academic degrees, which guarantee the appropriateness of the method used as well as the contents of a scientist's work. The time of distribution of new ideas, nowadays, is a couple of dozens of years. That reduction of time of distribution is mainly the result of the instantly appearing abundance of copies.

That apparently only quantitative difference, has to lead to deep qualitative changes in society, and eventually lead to shaping the system of education we know. The knowledge gathered in books is potentially available to everyone. All we have to do is to know how to read. The aspiration of the common knowledge of the art of reading and writing, appeared during the industrial revolution, as a result of a late reaction to the development of science, which was brought about by the Gutenberg's invention. After learning how to read (and write), people were finally able to demand to be led through the mazes of the world's libraries. Luckily, from the very beginning there was a group of scientists who knew the rudiments of knowledge, specifically the knowledge of physics, which were designed based on learning on mistakes, experimenting, blind wandering and systematic studies. Science was systematised and its particular branches were named. That knowledge was known, but because of how sophisticated it was, it was not 'easily adoptive' and the process always took time, sometimes even effort. Science was simplified to a certain extent, depending on how capable the listeners were, which was measured by the amount of knowledge they adopted to the point, and 
documented in various ways. That kind of a system of education was linear. Everyone started as an blank slate and was to walk a precisely defined path. The person could step off the path at any time and enjoy the knowledge possessed to that particular point. At the end of the path there was a point where all of the simplifications ended and then there were only the original ideas, which were written by scientists for other scientists. At that point, one could join them and become a scientist himself.

That system functioned successfully during XIXth century and until the mid XXth century. The question if the system could last eternally, is an unreasonable one. One might ask, how much longer could it have functioned. A linear system with the rudiments, canon of knowledge unfortunately leads to a situation, in which the new appearing elements of science are sooner or later being included in this canon, which is not bad itself because that is what progress is about, but the accumulated knowledge just becomes so vast that there are problems with one of the parameters of the system of knowledge, meaning the time needed by the candidate for a scientist to reach the stage when he becomes one. We reached a dangerously close distance to that border in the end of the XXth century.

\section{Electronic Age}

What is even worse, that was the time when the last division in the history of mankind by McLuhan (with modifications) took place: the Internet was invented. That medium, unknown to author of the "Gutenberg Galaxy", changed our reality in just ten plus years. The system of the functioning of the science changed as well. And what really happened? Yet again we deal with a change which is (only apparently) quantitative. By allowing the thoughts to be exchange through the Internet, the time needed for ideas to spread decreased almost to zero. A couple of hundreds of papers on high-energy physics gets to arXiv repository each day. Notification systems automatically send messages about the potentially interesting content to specific scientists. Not all of them, most probably only a negligible minority, check only even the titles of the works concerning their field. The situation is new. Nobody was prepared for that, especially the system of education. We are still cogs in the wheel of a machine which worked just fine, but in the XIXth century. Our actions are mostly based on textbooks from the Gutenberg Galaxy.

The boost in the epoch of information, when connected with the fact that even in the end of the pre-Internet epoch we were close to the border of efficiency of the system of knowledge (and education), makes us assume to believe that maybe we are just crossing that border, or are to cross it very soon.

\section{E. Education Crisis}

Obviously it may be denied and the reason for today's crisis of teaching exact sciences may be sought elsewhere. The examples are well known and there is no point of discussing such solutions as: 'more money for teaching resources and equipment is needed', 'the prestige of a teacher needs to be lifted', 'the lessons need to be more interesting', or finally: 'time and effort devoted for teaching science needs to be increased'. There were published multiple articles on those issues none of which resulted in some sort of a coherent solution. Furthermore, they could not do so, as the quantitative analysis of the process of storing and evolution of knowledge proves. The solution of the problem may be based on results, which are confirmed and solid.

\section{MODEL OF THE SYSTEM OF KNOWLEDGE}

In order to analyze the problem of the development of knowledge (science), its definition has to be given first. As E.P. Wigner [21] noticed long time ago, that is not an easy task.

It is quite easy to say that knowledge is the information given meaning and integrated with other contents of understanding [22], what is obviously true, but it does not clarify much.

The intuitive idea that knowledge is something more than information has led many authors to make distinctions between information and knowledge [23]. Some models treat knowledge as a higher form of information: the knowledge has to be extracted from the raw materials, and in the process, meaning has to be added to them (e.g., [4])

On the other hand the external reality we wish to study is available for the experimental studies only as percept and it cannot be experienced and recognized as such without the prior knowledge of some kind (e.g., [24, 25]).

One can measure the amount of knowledge using „knowledge assets" of Boisot, somewhat like Dawkins' "memes" [26], which exist in the heads of agents and survive by inhabiting the heads of as many agents as possible. (If they fail to occupy at least one agent's head, they die out.) This idea looks obvious for the oral epoch. Some kind of its generalization is used in the present work.

In the I-Space the new knowledge assets are created by agents (e.g., firms and institutions). This is the „microscopic" point of view. On the largest scale human knowledge is a self-organizing system [27] and the knowledge itself is organization, produced by trial and error, what makes the diffusive character of the I-Space solution so effective.

Leaving this problem for further considerations we would like to briefly introduce the rough, very approximate, but still useful concept.

There are two simple definitions of the actual state of knowledge the humankind possesses: one to vast and the other to restricted, none of which is satisfactory but both are worth to be quoted, because the truth has to lie somewhere in between.

The first is contents of all the volumes of all the libraries in the world. Assuming that an average book may be stored as an about $5 \mathrm{MB}$ computer file (100 pages, 50 lines per page, 100 symbols per line), the Library of the Congress containing about 30 million of books, 
handles about 150TB of information. The British Library with over 13 million of volumes, gives us the result of 75TB. Including other libraries all over the world and obvious repetitions (20-50\%) gives us more or less 250TB (of uncompressed) text data [28]. While reading one page per minute, it would take a man about a half of billion of years to read it all. A half of billion of years ago, the Proterozoic Eon ended and the oxygen which was newly released to the atmosphere, allowed the life forms we know to appear. There was nobody who could read then, as well as there was nothing to read. It is hard to tell how the world in a half of billion of years will look like. There is no certainty whether there will be anybody knowing how to read, and there is no proof if there will be anything left to read. So the existence of libraries does not mean anything, it does not mean everything at least.

According to the strict definition, the knowledge of the humankind contains only those pieces of libraries, which were familiarized with and are known to everybody nowadays. Something can be called ours, when everyone has it. Obviously, in that case our knowledge is extremely limited. That definition is not good.

So maybe it is enough to define knowledge, as all the information discovered and remembered by at least one member of the society. That proposal also has to be rejected, from a very simple reason: two different books often contain contradictory information and different interpretations, so the knowledge of humankind would be full of contradictions, meaning worthless in a big part. We wish it would be the other way round

Wigner finally proposed a rather flexible approach in defining the actual state of knowledge. It covers all the things the members of society might learn, if it came up to their minds, while there is a common belief, even certainty, that all of that forms a coherent image we call reality.

The definition fits the way the knowledge we previously described, is created. That knowledge may be measured in binary units - just as was described based on huge libraries. For other purposes, a more convenient method may be the analysis of the logarithm of that number, called negentropy. Even during the World War II, Erwin Schrödinger [29], a lecturer from Dublin Institute for Advanced Studies in Trinity College, noticed importance of that calculation for biological forms in general. Negentropy, calculated based on Wigner's definition of knowledge contents may play a similar role in refer to the civilization development. However, a more detailed analysis of that issue goes beyond this work.

\section{A. Oral Epoch}

We have to start the quantitative analysis, beginning with the epoch of the wise men. Development, which is basically the endurance of knowledge passed orally, may be described by means of a simple differential equation, describing a change in the quantity of knowledge as a competition of two different processes: extinction (let us call it forgetting) and creation. A specific model should be able to provide the stability of knowledge and resistance to temporary deviations. We propose the simplest solution in form of equation

$$
\frac{d N}{d t}=-\xi N+\alpha \beta N^{\kappa}
$$

$N$ stands for the amount of knowledge, $t$ is time, $d N / d t$ is a change of the amount knowledge in a particular unit of time, the rate of increase (or decrease, if the value is negative) of knowledge. First of the factors on the right side is negative and stands for forgetting. It is described in use of a one parameter only, $\xi$, which is defined as the inverse of the average time of vanishing of the particular information, in case when there is nothing which can reverse the process of extinction. In the epoch of the wise men, that time is about the lifetime of a wise man. Let us assume that it is one hundred years, just to make it simpler. The second, the positive element, describes the increase of knowledge. Parameter $\alpha$ stands for the number of people involved in the knowledge creation process. In the epoch of the wise men, the number was more or less constant. For a particular family, tribe, settlement, there were not many carriers of knowledge, and we may assume that it was a single individual. The second parameter, $\beta$, is analogous to the parameter $\xi$ describing forgetting, but is its additive inverse. It is the average rate of creating new stories. It can be both higher than the rate of forgetting, or lower, but it has to be beyond relevance in case of a properly functioning model. We may assume that the input in the resources of knowledge made by each wise man, based on one story, observation or discovery, contributes to $\beta$ around 0,01 .

There is also one more parameter on the right side of the equation $-\kappa$. It is essential to

ensure stability of the equation. If it was not there $(\kappa=1)$, then depending on the fact

if $\alpha \times \beta>\xi$ or $\alpha \times \beta<\xi$, the rate of increase of knowledge would be rapid and heading for infinity, or it would definitely decrease to zero. Values of $\kappa \neq 1$ ensure that the value of $N$ has to reach stability after some time, which is determined by the solution of the equation

$$
\frac{d N}{d t}=-\xi N+\alpha \beta N^{\kappa}=0
$$

as

$$
N_{\text {stab. }}=\left(\frac{\alpha \beta}{\xi}\right)^{\frac{1}{1-\kappa}}
$$

Fig.1 illustrates that situation. It is visible, that in spite of all the variations we deal with, after some time (a few generations for the parameters of the given model) the stability is restored again. As long as the role of the parameter $\kappa$, shown in Eq.(3) and illustrated in the Figure, is clear from the mathematical point of view, its interpretation and meaning in the real world requires an 


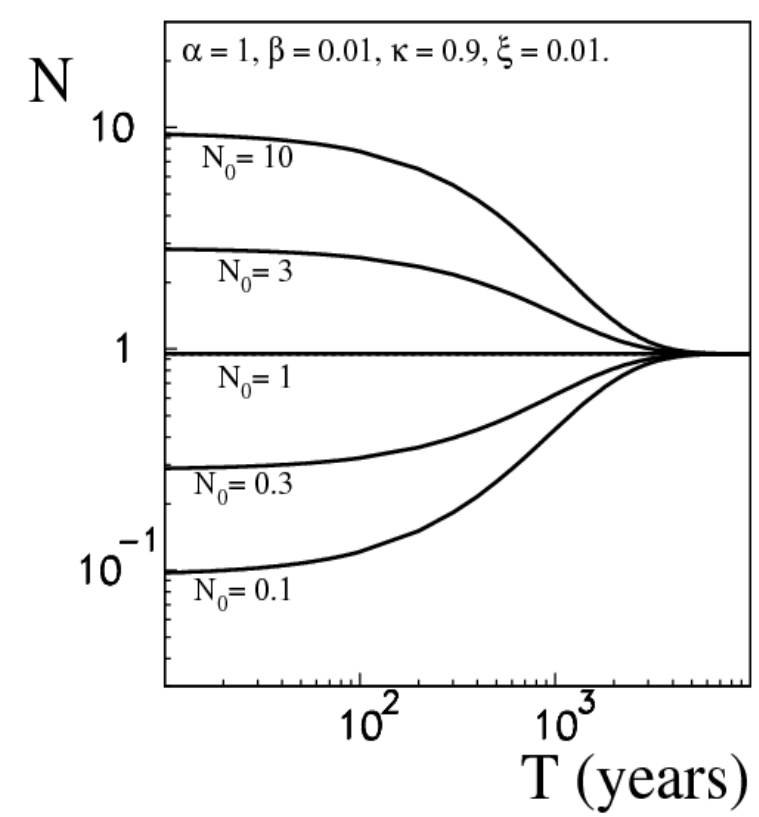

Figure 1. Evolution of knowledge $(\mathrm{N})$ in the oral epoch, for the typical parameters with various initial states. As may be observed, in spite of the variations, the state of knowledge stabilizes in the long run.

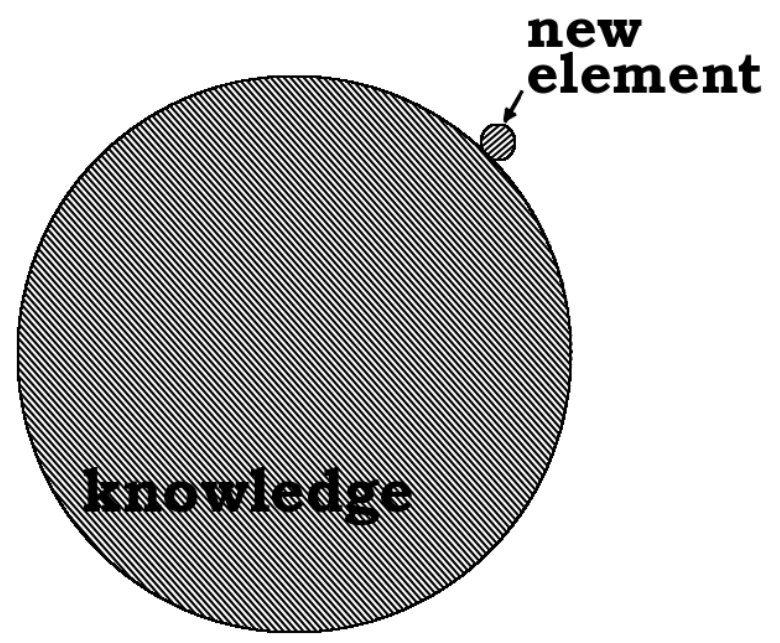

Figure 2. The scheme of increase of knowledge in a model of a snowball.

additional comment. Understanding why the pace of the knowledge creation is not simply proportional to its resources but 'slightly less' proportional, is not something obvious. The 'snowball model' explains that situation (Fig. 2).

Let us imagine knowledge as a set of points, for instance as a plane figure. If someone is fond of symmetry, lets make it a circle. Each fragment of the circle is a peace of knowledge, and someone who knows it all, meaning a wise man, may try to add some element do the possessed resources. That element is then added to the edge of the
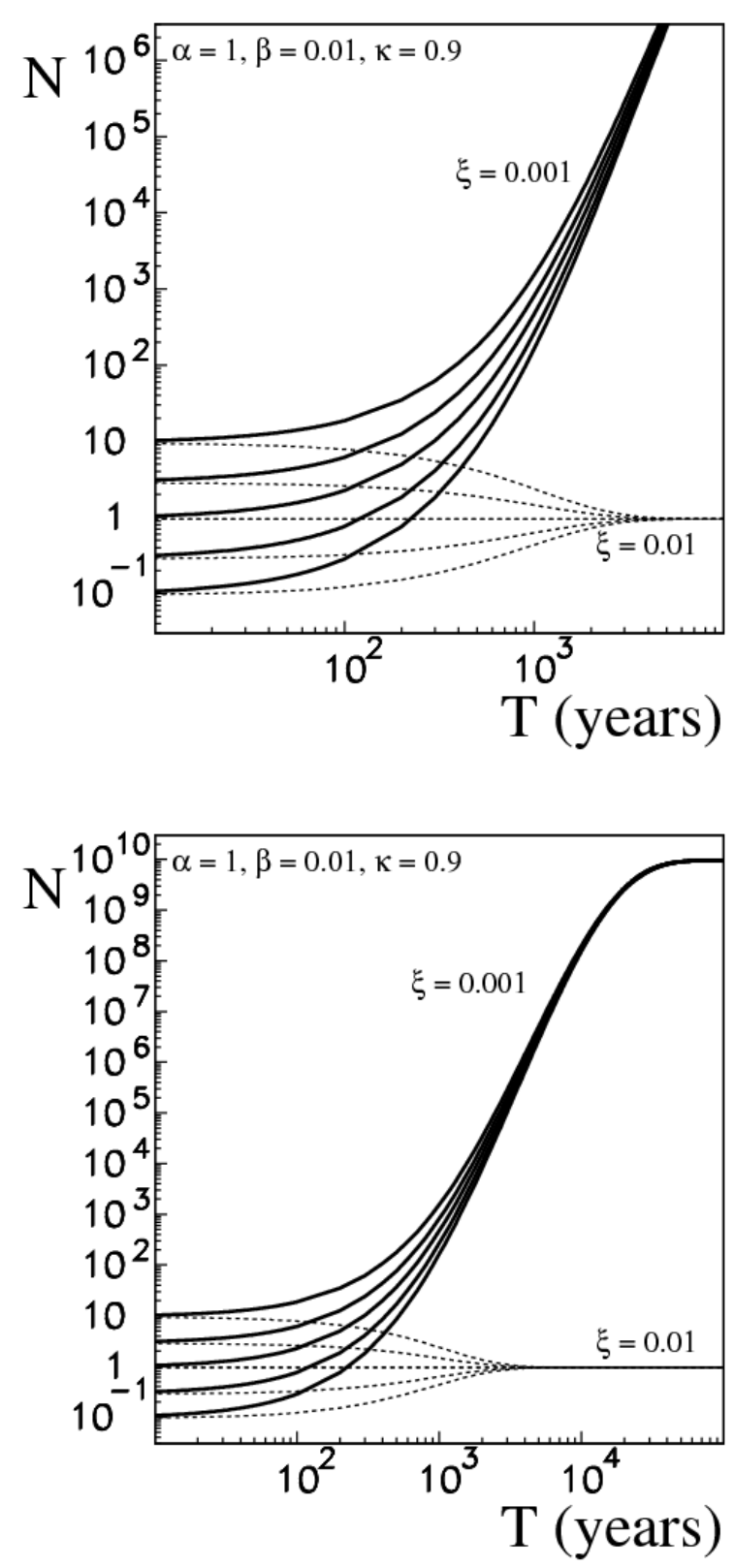

Figure 3. Almost exponential growth of the value of knowledge, when the parameter of forgetting is increased by 10 (top picture), and it reaches asymptotic value 10,000,000 times bigger than for $\xi=1 / 100$ (bottom picture). Curves from Fig. 1 are presented for comparison.

possessed knowledge. In the same way a rolling snowball grows. The amount of space on the edge in proportion to the capacity (length) of a two-dimensional circle $S=2 \pi r$, and, because the amount of space within the circle is $V=\pi r^{2}$, one get $S \sim V^{1 / 2}$, in the case of a threedimensional sphere it is $S=4 \pi r^{2} \sim V^{3 / 2}$, and so on. In case of knowledge, there is no point to limit the number of dimensions. Yet, if it is finite $(\boldsymbol{n})$, then the proportion of the edge and the inside, which defines the index $\mu$, will always lead to result which is less than $1(\kappa=(n-1) / n)$. 

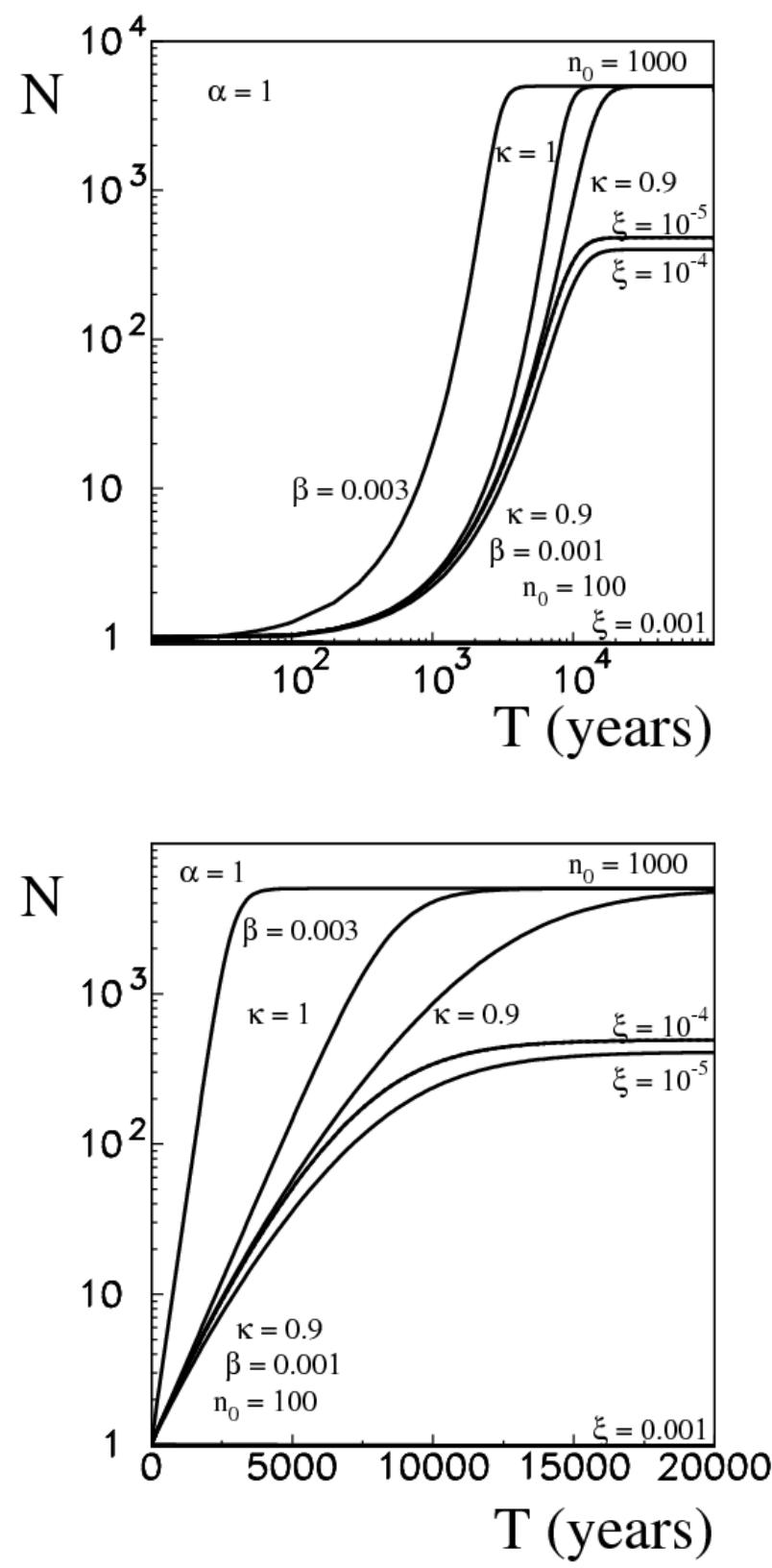

Figure 4. Knowledge development in the epoch of the written word (in both: logarithmic and linear evolution time scale).

\section{B. Manuscript Epoch}

Introduction of the first scientific revolution: invention and application of writing apparently does not require any significant changes in the initial Eq. (1). At first glance it may appear that the only result is a significant increase of 'persistency' of knowledge and all it takes is to decrease the pace of forgetting of knowledge and the model will still be correctly describing the new situation. The endurance of the symbols engraved on walls, horns, bones, imprinted on clay, painted on a wall or a piece of leather, is much longer than in comparison to the fragile brain tissue. If it is at least ten times longer, then the influence of the element of forgetting gets much lower. The closer the value of the index $\mu$ gets to 1 , the less significant is the factor of forgetting, what results from equation Eq.(3). The limit value is a number certainly greater than 1, to a high power. The solution of simple equation Eq.(1) gives an almost exponential growth, which reaches absurd (meaning infinity) very quickly. Those results are illustrated on Fig. 3.

In the case of our definition of science, the obvious thing is that its volume has to be limited. The level on which we have to stop is not relevant yet, but we certainly have to complement the initial equation with a inhibiting factor, but the factor would have to be found in the real world of the epoch of libraries first.

The previously proposed model of an increase of knowledge, the model of a snowball, beside the fact that it substantiates the existence of an index which is less than 1, it also suggests the correct solution of this limit problem as well. New knowledge appears, as it was already mentioned, and it is added to the regiments of knowledge only if it 'fits' its existing profile. Thereby it is wise to assume that the creation of a new element of knowledge requires getting acquainted with its existing state. So every scholar has to spend some time on studying, before he discovers something new. That model is rather accurate and it would be hard to convince anybody nowadays (maybe except the ambitious pupils from primary schools), that it is possible to achieve something really smart without learning anything previously. On the other hand every scholar has a limited time for learning. If the time of learning approaches the time of living of a scholar, then only the long-lived individuals would be able create new science. It obviously sounds irrational, but unfortunately that process sets the limit for the development of science out of necessity (especially for particular fields of science).

In order to write it down according with our primary considerations, let us assume that the discussed relations are in the simplest, linear form. An additional factor which the rate of creation has to be multiplied by, depends linearly on the time left for the scientist after his graduation, for creative activities, and the more knowledge is there to be learnt, the less time he has. The modified equation is now in a form:

$$
\frac{d N}{d t}=\xi N+\alpha \beta\left(1-\frac{N}{n_{0}} \frac{\tau_{0}}{\tau}\right)_{>0} N^{K} \approx(\alpha \beta-\xi) N-\left(\frac{\alpha \beta \tau_{0}}{n_{0} \tau}\right) N^{2}
$$


There are three parameters describing approaching the saturation point: $\tau_{0}$ - the time needed to comprehend a one unit of knowledge, $n_{0}$ - the measure of that knowledge unit, which is, in fact, only setting the scale on the vertical axis, and $\tau$ - the average lifetime of a scholar.

Those three parameters are used in equation Eq.(4) combined, so there is no point to qualitatively reflect on each of them separately. In all the calculation below, we assumed that $\tau_{0}=1$ and $\tau=100$, As one can see, the equation became non-linear (even if we assume $\kappa=1$ ) and its solutions for different values of parameters are shown on Fig. 4.

There is a visible change of the increase of $N$ depending on the parameter, which describes forgetting knowledge. There is also a visible relation with the value of the index $\kappa$, in case when it is significantly different than 1. Which is quite obvious. A lower index means slower increase of knowledge, however there are now no contraindications for assuming the limit value of 1 .

The dependence on the value of $\beta$ is also obvious. That parameter describes how active a scholar is, and the higher activity the higher increase of knowledge. The asymptotic value is achieved for big values, just as we wanted it to. It is specified by the parameter $n_{0}$ (combined with $\tau_{0}$ and $\tau$ ), which describes the amount of knowledge a scholar can comprehend. For most of curves on Fig.4, was assumed to be $1 / 1000$. In case of the epoch of the wise men, $\beta$ was previously set to $1 / 100$. It seems to be justified, because creativity of 'the scientists' from the epoch of no writing, had to be big enough to ensure the continuity of knowledge. In the moment when knowledge could be written down, meaning more enduring, the importance of creativity decreased. That parameter is not critical for our discussion, because it is always multiplied by $\alpha$, which describes the number of people who are involved in the process of creation, which is now assumed as a constant, what is not consistent with the growth of population and the development of public system in the epoch of libraries. We will return to that issue later on.

\section{The Gutenberg Galaxy}

Analysis of the growth shown on Figs. 3 and 4, we may see that the picture is not satisfying yet. If we assume that the epoch of writing started in $500 \mathrm{BC}$, (not to mention what the results would be if we agreed to set it as $20000 \mathrm{BC}$ ) then close to its end, meaning after 2000 years, we should be able to observe a significant exponential growth of libraries, independently of the date we set (within limits of reason). Maybe there actually was a similar situation in the end of the Middle Ages, but it is highly unlikely for a situation like that to occur much earlier, but curves of increases which represent the solution of Eq.(4) are exponential from the very beginning. The increase of knowledge observed over the centuries, seems to be more linear than exponential. In order to make it so, we have to introduce a one more modification, which is the last one for now.

As it was previously mentioned, the knowledge distribution process in the epoch of the wise man, was in
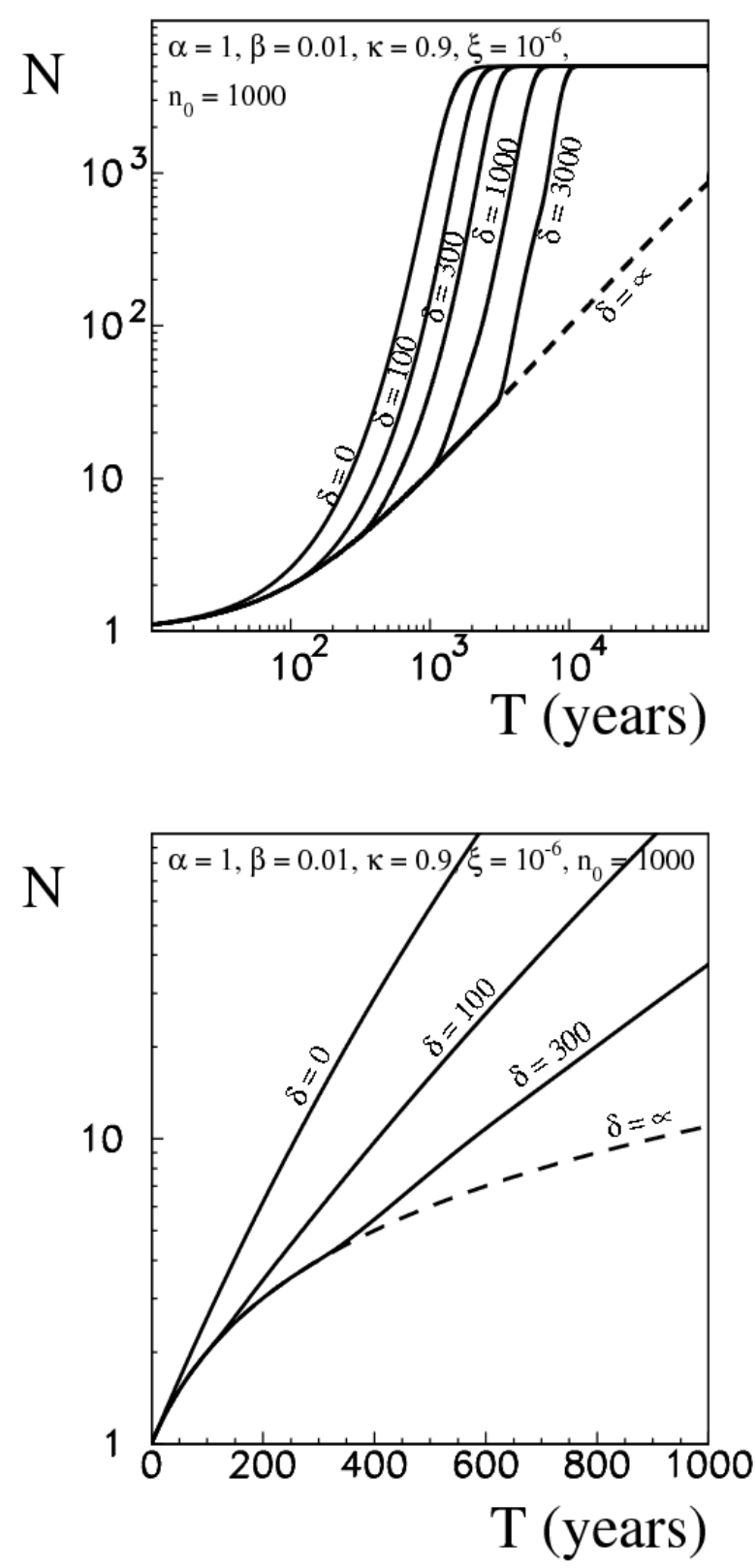

Figure 5. Development of knowledge in the epoch of the written word, depending on the rate of proliferation (time of delay) of new ideas. Dashed asymptotic line stands for the constant increase of knowledge. The solid curves present the acceleration caused by the increase of knowledge assimilated after time $\delta$.

fact rapid. An invented story, which was then told to people (meaning the whole social group: family, clan, tribe), almost instantly became well known. A scientific discovery, or whatever we call it, 'came into effect' without any unnecessary delay. The situation changed drastically when the civilization came to a point when the knowledge needed to be reprocessed to symbols, then encoded and preserved. That encoded knowledge went around in the form of copies, but it did not reach far because of its high value, so that scholars had to travel in 
order to gain that knowledge, and that process took time because the interested people had to visit many places in order to learn this or that. The duration of the knowledge re-distribution process may be variously assessed. The good example is how the 'world' familiarized with the works by Aristotle, starting with XIth century and, than after its rediscovery in the World of Latin or the Copernican Revolution which took place in 1543 (however from our perspective, maybe the date of the beginning of writing would have to be set even 40 years back). It was not a short time, in the second case Galileo, one hundred years after Copernicus, still could not feel safe at the Tribunal of the Inquisition, and Newton wrote "Principia..." which gave some sort of an explanation of the forces which applied in the planetary system, and that was another one hundred years later. It has to be remembered that the fate of other discoveries with less spectacular consequences, as in less important, might have evolved even slower.

The effect of delay in distribution of the new knowledge may be included by introduction of a new parameter $\delta$. It is defined as the time from the very introduction of a new discovery, until the first effects it may cause in the system of knowledge.

$$
\begin{aligned}
& \frac{d N(t)}{d t}=-\xi N(t)+ \\
& +\alpha \beta\left(1-\frac{N(t-\delta)}{n_{0}} \frac{\tau_{0}}{\tau}\right)(N(t-\delta))^{\kappa}
\end{aligned}
$$

The solution of that equation is illustrated on Fig. 5. Both graphs present the same relations, but with a different scale in refer to the axis of time. The family of continuous curves shows the results for different values of the delay time $\delta$. The asymptotic dashed line stands for the increase of knowledge in case of infinite delay. In such case, the newly invented ideas do not influence the rate of increase of knowledge. The remaining cases show, that the delay not only influences the point at which that particular curves leave the asymptotic line, but also influence their slope, which stands for the rate of increase.

The value of the knowledge obtained over a very long period of time is constant, however the value is no longer defined by equation Eq.(2), but

$$
(\alpha \beta-\xi)-\left(\frac{\alpha \beta \tau_{0}}{n_{0} \tau}\right) N=0
$$

\section{THE PRESENT SITUATION}

Having what seems to be a complete equation describing the development of knowledge, we may attempt to apply it in a particular case of humankind as such. The values of parameters which were described for all of the four mentioned epochs, are presented in Table I.
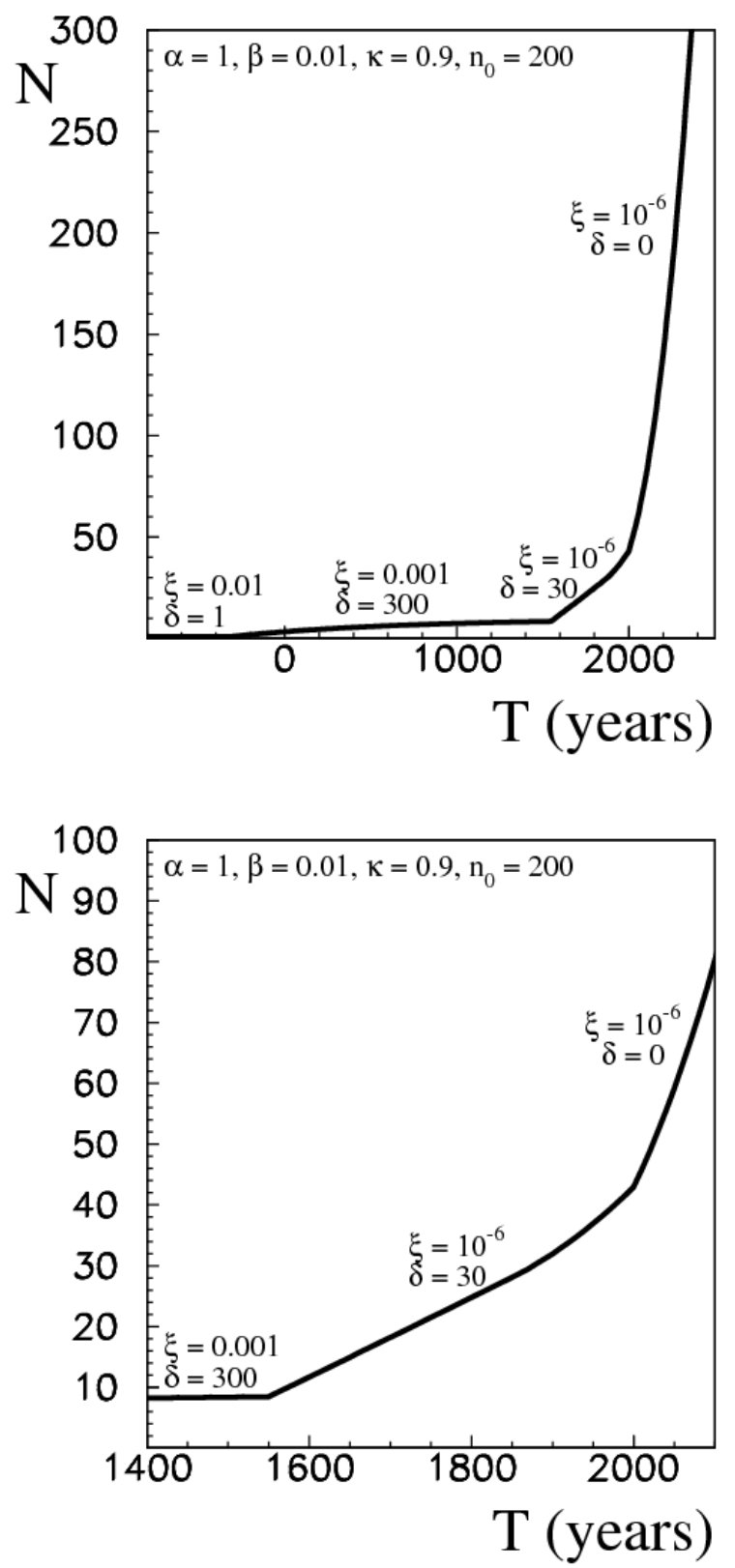

Figure 6. The history of knowledge of humankind in all of the four epochs, with reasonably adjusted values of the parameters. The picture on the right is the magnification of the fragment concerning the XV-XXI centuries.

The introduction of printed books increased the circulation of information ten times and at the same time caused that once a book was printed, then it stayed in the circulation forever, or at least lasted in it as long as anybody wanted to read it. The current epoch, the epoch of the Internet caused that the time of proliferation of knowledge decreased almost to zero. Those changes were discussed in details in Sect. I. The values proposed in Table I are not adjusted precisely, but they are rather the 
TABLE I: The values of parameters of the model in particular epochs.

\begin{tabular}{|r|c|c|c|r|c|r|}
\hline epoch & period & $\alpha$ & $\beta$ & $\delta$ & $\mathrm{K}$ & $\xi$ \\
\hline oral & $-500 \mathrm{BC}$ & 1 & 0.01 & 1 & 0.9 & 0.01 \\
\hline manuscript & $500 \mathrm{BC}-1500$ & 1 & 0.01 & 300 & 0.9 & 0.001 \\
\hline Gutenberg Galaxy & $1500-2000$ & 1 & 0.01 & 30 & 0.9 & 0.000001 \\
\hline electronic & $2000-$ & 1 & 0.01 & 0 & 0.9 & 0.0000001 \\
\hline
\end{tabular}

values which seem to be reasonably close to the truth. They are close enough to make the solution comparable with the observations of the actual development of science over the centuries. The value of the parameter $\alpha$ will be discussed in the following. For now, its value was set equal to 1 for all of the epochs, so the presented result may be interpreted as the amount of knowledge per a single individual. The results of Eq.(5) for the given parameters are shown in Fig.6.

The horizontal axis on Fig. 6 was matched to the usual time convention. As it is presented on the left picture, the invention of writing did not trigger any sort of a revolution, the Gutenberg's invention resulted in both scientific and technical-scientific revolution, visible as a fast but more or less linear increase in the XVIIth century, expressed clearly in the right picture.

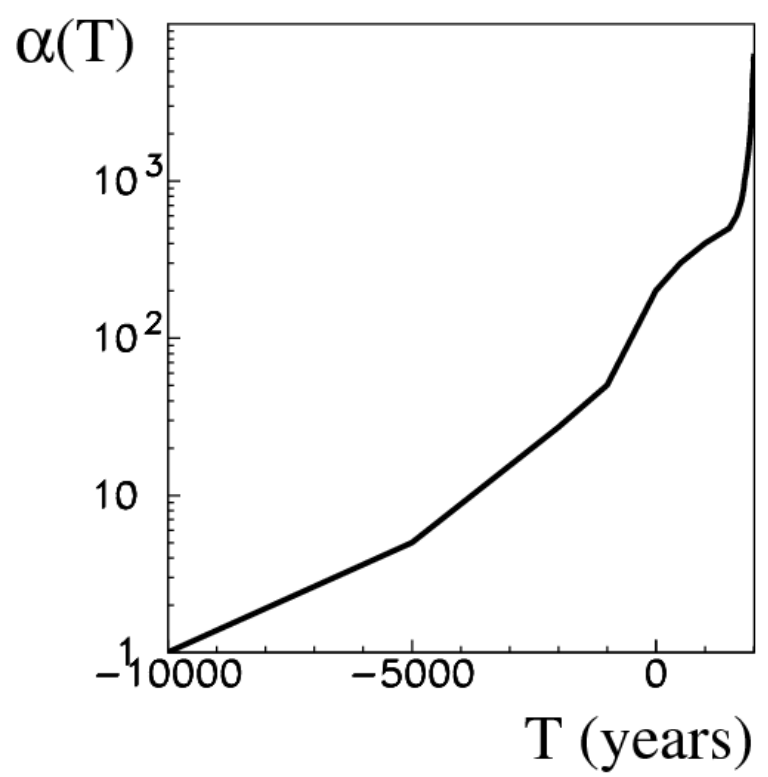

Figure 7.The population of Europe (in millions) over the centuries [30].

What is the most interesting from our point of view, is the beginning of the current (and future) epoch, originating at the turn of centuries and caused by a sudden increase of the flow of information.

\section{A. Population growth}

There is a one more modification of the model left, meaning a more appropriate match for the value of the parameter $\alpha$, to be more specific. As it was previously mentioned, it is related to the number of the people involved in all the intellectual activities of a given social group, which is more or less the number of the people in the 'world', and by world we mean the world which was available to an average individual, a scientist particularly. We can estimate the increase of the population over the centuries rather precisely. It is presented on Fig. 7. By introducing value of the parameter $\alpha$ being proportional to the population of Europe, we get the conclusive image of the evolution of science from the very ancient times, with extrapolation for the future. It is presented on Fig. 8.

It shows the boom of the epoch of the Internet, in total. Reaching the point of saturation occurs more or less after 30 years. The properly adjusted values of parameters may obviously alter that period, nonetheless the fact is that whatever our approach to that issue may be, probably now is the time when we are inevitably nearing that sad moment, however it is also probable that it has already skipped our attention.

It may apparently seem that reaching the state of stagnation, stabilization of the value $N$, should have been noticed and lead to countermeasures, or at least astonishment. However it is not so. Let us remember that, for the whole time, we examine knowledge concerning one field only, meaning theses, statements and theories that are related and form a coherent unity. The upper limit originates from the fact that in order to reach further, one would have to comprehend so much information, that it would exceed his life time to do so. In such a situation, and obviously before that, there must some concepts appear, based on a partial familiarity with the state of knowledge of a particular field. If these are not full of nonsense, obviously incorrect, missed, banal etc., then they are independent from some parts of knowledge gathered up to now. If over time there would other ideas based on that new concept appear, and the construction of a system of knowledge around them starts, a one which would only partially be consisted with the previous rudiments of knowledge, then a new field of knowledge would appear. It would start to develop and spread just as same as was in case of the parent field until then. Primarily less wealthy and more humble, it allows the scientists to prove themselves in the new field, as they did 

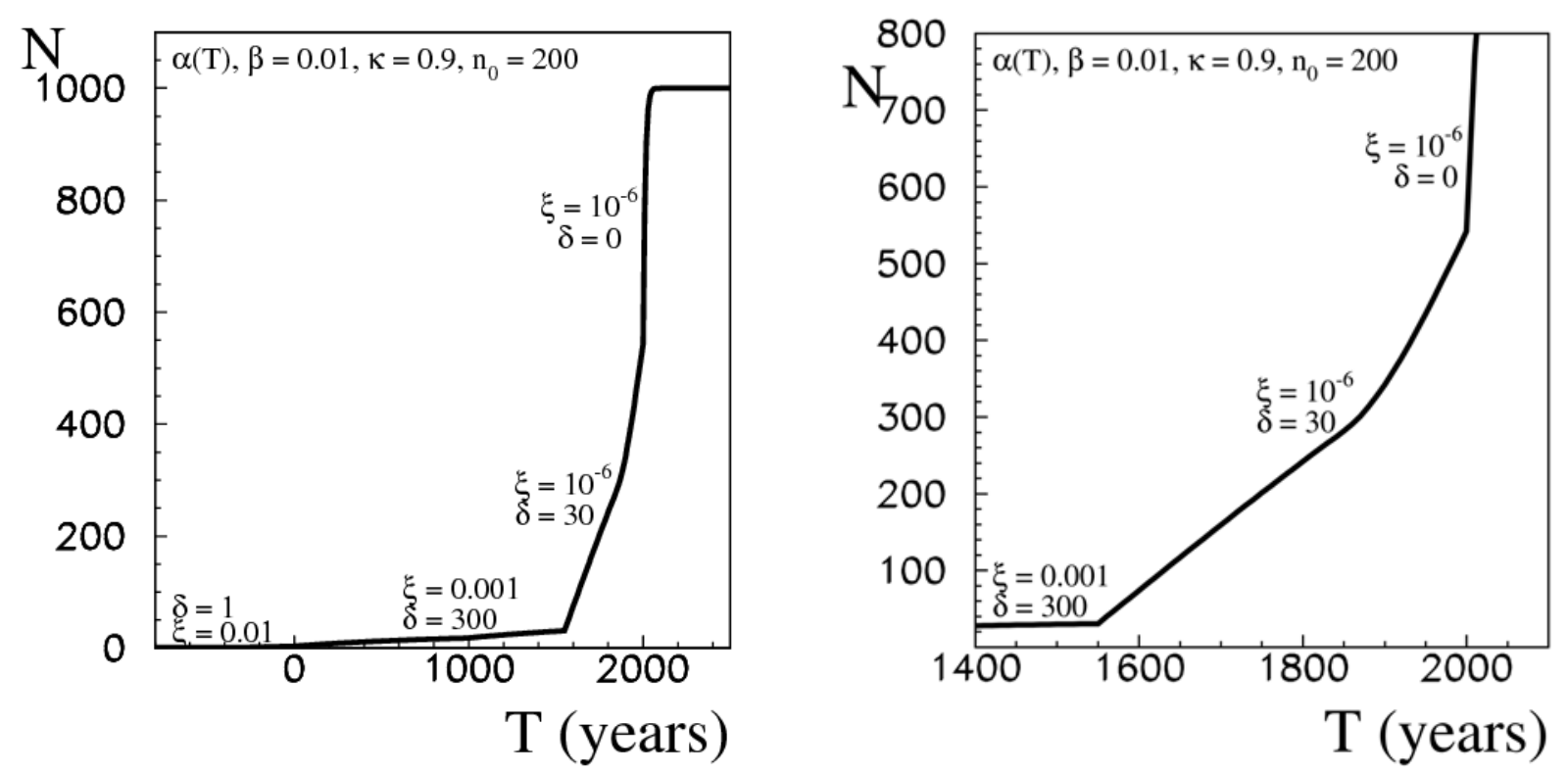

Figure 8. The history of the knowledge of the humankind in all of the four epochs, including the demographic growth from Fig. 7. The picture on the right is the magnification of the fragment concerning the XV-XXI centuries.

not have opportunity to do it in case of the old one. The increase of the new field is according with Eq.(5) and the parameters which are proper for a given epoch. At the same time nothing limits the old the fully grown systems, and new fields and specialities may emerge from them. All of which develop more or less with the same pace. The possible differences may come from differences concerning the parameter $\alpha$, which describes the social attractiveness and the fact of a particular field being 'scientifically' fashionable. The more fashionable fields, develop faster.

The quantitative analysis of that process leads to an identical equation, in terms of the general idea, as Eq.(5), where the value of $\mathrm{N}$ would no longer stand for the amount of information, meaning available knowledge, but the number of existing fields of science. The interpretation of parameters of such a model will obviously be different. However, the solutions will be similar to those presented above. The number of fields will be snowballing which does not seem to be good.

The process of division of the system of knowledge to fields which are significantly independent,

is not a new phenomenon. The study of inanimate nature, the changes within it as well as meaning of those changes, which basically is all that we call sciences, was all included in ancient physics. Nobody wonders that nowadays we have astronomy, chemistry, meteorology and biology. That division, which is obvious and results from the described way of creation of new fields, leads to specialities. Nowadays, it is hard for a botanist to communicate efficiently with a nuclear astrophysicist. Those two fields have so little in common, that the newest inventions in both fields are totally independent and have nothing in common. The process of division of science goes so far that for instance within physics, a specialist of quantum optics is not able to discuss much about his work with a cosmologist, if he wants to go into details. People like Boyle and Smith, the winners of the Noble Price in Physics in 2009, might be able to understand the importance of the works of this year's laureates Geim and Novoselov, but there are probably not many people in the world who could actually explain what exactly all of them were awarded for. Disciplines, fields and specialities of modern physics have little in common, and the examples are numerous. All of that implicates that the process of snowballing of specialities has already started.

Discussing the expected final state of that process, if there even is one, is out of the scope of this paper. We would like to ask a question though, whether the state that was described above and the perspective of its evolution would influence the system of education or not, and because of the fact that the answer to that one is pretty obvious, we need to rephrase the question to the form: what is the influence and how to deal with it?

IV. THE PARTICULAR APPLICATION: THE CRISIS OF THE (SCIENCE) EDUCATION SYSTEM.

The system of education is to prepare the youth to move around the world efficiently, and without collisions. We may also ambitiously assume, that we would like the world to become more and more friendly. In order for it to be so, it would be good to know it first, and then to make it better as much as it is possible. The history taught us that the attempts without understanding the world, or even against that, led to some unpleasant consequences. Assuming that those postulates are true, we come to the conclusion that the knowledge we possess now, needs to be passed to the youth, as knowledge meaning wits lets us to understand those things which are around us in use of imprecise terminology. The times when it was possible to 
teach someone everything, before he gets too old to use that knowledge, are definitely long gone. It was probably figured out back then, that the knowledge of a particular field needs to be put in order. During those times, there were only a few fields of sciences, and by saying a few we mean about one, so there is no difference if we talk about teaching physics or science in general. Science need to be systematized first and then their basic rules have to be systematically and holistically compiled in form of books, in which each chapter would describe a particular field from the very basics and go into details, whereas the limit of those details would depend on the purpose of the book. Next, those books would have to be extended until they reach the wanted volume, and the extension would need to be even in refer to all the fields. The volume we spoke of, was measured by the amount of time of teaching, including the age and predispositions of the student. Those kinds of books were adjusted to the general teaching scheme and met the criteria of equality and universality. A student could stop the process of education at specific moments and then he was left with the knowledge he acquired up to this point. The most determined students would acquire the status of scholars and specialists of a given field, academic degrees and titles. By design, they would know a particular field of science. That design worked well a hundred years ago, but nowadays the statement could be confirmed only by some radical optimists.

According to the analysis of the process of increase of the knowledge resources, as a great number of new fields and specialities arises right in front of us, the system crashes. The reason is simple: the scheme which was designed in the beginning is not sufficient enough. It cannot cover everything that was invented and is important from the point of view of knowledge of the humankind. Among other reasons, the most important is that nobody knows everything. Nobody knows the whole knowledge concerning all the fields of physics, and if such person still exists nowadays, he will cease to exist very soon. Collegial bodies may be appointed, the attempts of what are being undertaken. They may create some sort of a scheme, but it would be a compilation and a comprise, so it would basically be something different than it should be. The canon of knowledge compiled in that way become some vast, that after the previously described process of simplification, it no longer includes anything but the unimportant set of the arbitrary basic terms and rules, the true meaning of which may only be discovered on some advanced stages of education, after getting acquainted with multiple terms and rules.

The only solution of this situation is the rejection of basic principles of common, general need of teaching of physics/science.

The rejection of the present commonality of education and replacing the old system with the one will result, particularly during the transitional period, in lack of the so called 'specialists', who are those people who by choosing a particular profession would want to, or even are obliged to, possess the knowledge of physics which was defined according to the present standards. The need of those professions will decrease in the long run, however there will probably always have to be a group of people like the one mentioned, who after receiving the general education, would educate themselves in exact sciences as well, according to the pattern above. They will choose the career path which requires particular skills, meaning the use knowledge of exact natural sciences. They will be required to get through the physics course, similar to the one at the advanced GCSE level. Obviously the contents which are to be taught should be narrowed, in order for the knowledge to present some sort of a standard. There has to be a possibility of elimination, or inclusion to the program of the whole themes, if needed. Leaving those kinds of choices to the teacher at this level, drastically increases the meaning and the prestige of a teacher. Due to the fact that this way of education is the most similar to the current one, it will be the easiest to be introduced. It requires only to reconstruct the base of the program, and involve some changes in contents of particular themes.

The third way of education concerns those people who intend to become 'the wise man' in the future, meaning those people described in the beginning of this work. Nowadays, they would have to be called scientists. The people in the future will try to increase the knowledge resources of the humankind, they will attempt to know the unknown and undiscovered. In order to do so, a clear image of the whole is needed, different for everyone, but it has to be significantly deepened. The knowledge passed should be as deep as possible, however one has to bear in mind not to overstep with the formal perception of the taught contents. It is not always possible, but the attempts should be undertaken. This way and the previous one, are divided by the way which was designed for the specialists of applied sciences. A future scientist might solve the problems which are included in the typical book of problems nowadays, but does not necessarily have to know how. Obviously it is not true that he does not have to learn how to solve problems, but the main point is that the nature of the problems would be different. Methodology of physics, as a science seeking for answers but also questions that nobody can answer to, is something qualitatively different than searching for solutions of standard problems. Someone who chose that way, should acquaintance himself with that methodology. Modern schools do not provide that. The best student can solve complicated problems which were maliciously compiled by the professionals whose job is to compile those problems, whereas the problems with face in the real life rarely are malicious, but they often are hard and do not have to be typical. The system of education specifically designed for that group of the young people, practically does not exist nowadays. All of the actions concerning the so-called gifted youth, are rare. As a course of time, we may expect the increase of pressure concerning that issue and even now it is high time to start wondering how to pass the knowledge needed by the youth to create new values (scientific). The easiest way is to stall, meaning stuff the hungry minds with useless skills, some abstract mathematical calculations, which 
may appear to be useful one day, and to wait until they graduate. After that they get caught in the web of higher education, which tends to operate more and more similarly to the previously described system. Making the institutions of higher education responsible for teaching the gifted youth results in losses, from which in many cases they cannot recover from. The more absorbent and flexible minds undergo the process of conformism, which is when their potential growth of hidden capabilities and creativity suffers. On the other hand it is hard to organize classes in the way it would fit the most ambitious individuals, considering only school and its little capabilities. The third way has to be conducted together with universities, colleges and academies, or other institutions where there is even a potential possibility of encountering science in its form in statu nascendi. In order to learn how to create new values and methods of using the current, not only intellectual, tools but also the instrumentation which is close to the current one, the youth has to leave the school buildings, because it is not possible to create modern laboratories of various fields there (even physics lab). Moreover, it is futile. Another possibility is to visit university labs. In this way, the postulate of modernity and direct access will come true, but it will automatically introduce the unwanted element of selection based on the distance from the place one lives to the leading science centers. Those limitations are hard to overcame, so basically it is not possible to base the third way of education on that. The only option seems to be to create some small educational laboratories with on-line access, in the vicinity of the big science labs at university compounds. In use of those modern technologies, the young people could conduct classes from anywhere and essentially study and search for solutions of the problems of particular interest to them, not limited by local resources, using both the base and the help of the academic scientists.

There are even more demanding challenges which a teacher on the third way of teaching has to face with. He has to be the guide for the youth in their search, he has to play the role of a medium between them and the facilities which share their on-line labs, but he also has to know how to solve problems. On the other hand, he is not required to possess some specific knowledge of physics, because that may always be asked for, providing one knows how and who to ask. As a course of time the students themselves have to learn how to ask proper questions, however it is the teacher who should show them how to do it first. The teacher should coordinate the work of his students at that stage, propose some directions of enquiry. How active he is needs to obviously depend on the group he works with. Those groups will not be large, because they cannot be. Irrespective of the way we define 'particularly gifted students' in physics, we cannot expect that it exceeds 1 per mille of the population. Organizing a lesson for such a small group is a problem, however for the same reason those groups will be cared for by the teachers with particular predispositions, skills and training required for that kind of a work with the youth.
The involvement of universities, colleges and academies in the teaching process at the pre-academic level, seems to be particularly valuable for, at least, a couple of reasons. First of all, the institutions of higher education may share, on-line, a part of their unique equipment, they have a capable staff at their disposal and potentially are ready to provide the substantial help in solving complex problems which may occur during classes, and finally, help in staff training. On the other hand those institutions should have interest in finding individuals who are interested in science, potentially gifted, who may constitute to the development of those institutions in the future.

\section{CONCLUSIONS}

The 'three-way system' which is described above, may introduce the society of the $\mathrm{XXI}^{\text {st }}$ century to the world of modern science. It provides an image of a rational reality to everybody, what gives basics for the scientific view of the physical world and understanding the laws of nature. It allows the development of the technical fields, as well as the relative ones, allowing to show the path of education for the people who want to become the trained staff in sophisticated fields, new technologies, with skills which require specific knowledge. It also gives the opportunity of development for potential creators of science, allowing them to possibly early try to face with the challenges of science.

In comparison to the XIXth century system, the role of a teacher in the educational process increases. The teacher becomes the creator of the teaching program. He implements, so he needs to be prepared for that, what means that the process of education for teachers has to be altered. The teacher cannot be a student of physics no more, meaning a one who wrote his master thesis concerning "the study of dynamics of particles in the reaction chambers of zeolite in use of the methods of nuclear magnetic resonance relaxation of deuterons", or "the neutron-excess nuclei structures of double magic ${ }^{208} \mathrm{~Pb}^{\prime}$ (the real titles proposed for the academic year of 2010/11 in one of Polish Institutes of Physics), and attended some pedagogical courses.

When leading students the first way of education prepared for general, wide audience, it is not relevant for the teacher to possess deep knowledge in one and a very specific fragment of the knowledge of physics. The teacher needs a broad general knowledge and he should acquire it during his higher education on the speciality of teaching physics, both in theory and in practice. This kind of studies is at least of the same, but probably bigger importance for the society as sophisticated theoretical, optics, solids state or high energies physics which could lead to the Noble Price, in principle.

The program of university studies for teachers must also contain the training concerning the teachers, who are to work with the young people interested in knowledge, and particularly interested in physics concerning the second and the third way of the proposed education system. The narrow specialization is irrelevant, or even 
harmful here. The efficient capturing of basics of physics is necessary for a proper training of the future specialists, for instance at the technical universities, for specialists in chemistry, physics, or even in teaching.

The education for teachers concerning the third way is the most debatable, as on one hand the narrow specialization would result in easier management of the students, once they were to follow the same direction. However it has to be assumed that, as a general rule, it will not happen that way. The specialists on the third way may be a part of the university staff, and there job is to deliver the necessary knowledge concerning the topic they offer, to the teacher. Instead of that, the teacher should master the art of preparation and conducting experiments, making observations, analyse the results and then conclude. Those skills are extremely important not only for physics, the lack them sometimes leads to unpleasant misunderstandings, particularly if it is about the statistical analysis and general knowledge of statistics.

The requirements of the proposed system, in refer to teachers, are high and complex, but it seems that it is possible to include them in a system of education, what would make it coherent. The task is not easy, but necessary to be completed because only the trained teachers are able to painlessly lead physics and sciences education out of the crisis, in which they undoubtedly are, as it was proven.

\section{SUMMARY}

We have shown that it is possible to find one sample equation describing the development of the knowledge/science on a global scale. In principle the method can be used to establish the model for microscale, the kind of the I-Space picture, to have a detail insight into the mechanisms of knowledge creation of distribution in time.

We have used it to estimate few parameters determining the evolution of the humankind knowledge as a whole and predict the near future. This can be useful in searching for remedies to the observed worldwide science education crisis.

\section{REFERENCES}

[1] I. Nonaka, The knowledge-creating company, Harvard Business Review, 69, 96 (1991)

[2] I. Nonaka, A dynamic theory of organizational knowledge creation, Organization Science, 5, 14 (1994)

[3] I. Nonaka, P. Byosiere, P.C. Borucki, N. Konno, Organizational knowledge creation theory: a first comprehensive test, International Business Review, 3, 337 (1994).

[4] I. Nonaka, H. Takeuchi, The knowledge-creating company. How Japanese companies create the dynamics of innovation, Oxford University Press, Oxford (1995).

[5] I. Nonaka, N. Konno, N. (1998) The concept of „Ba”: building a foundation for knowledge creation, California Management Review, 40, 40 (1998).
[6] I. Nonaka, R. Toyama, P. Byosiere, A theory of organizational knowledge creation: understanding the dynamic process of creating knowledge, in: M. Dierkes, A.B. Antal, J. Child, I. Nonaka, (eds.) Handbook of organizational learning and knowledge, Oxford University Press, Oxford, (2001).

[7] I. Nonaka, R. Toyoma, Why do firms differ? The theory of knowledge-creating firm, in: K. Ichijo, I. Nonaka (eds.) Knowledge creation and management. New challenges for managers, Oxford University Press, Oxford (2007).

[8] C. Bratianu, A Critical Analysis of Nonaka's Model of Knowledge Dynamics, Electronic Journal of Knowledge Management 8, 193 (2010).

[9] O.K. Harsh, "Three dimensional knowledge management and explicit knowledge reuse", Journal of Knowledge Management Practice, 10, 1 (2009).

[10] M.E. Nissen, Harnessing knowledge dynamics. Principled organizational knowing and learning, IRM Press, Hershey (2006).

[11] D. J. MacKinnon, R. E. Levitt and M. E. Nissen, Modeling Skill Growth and Decay in Edge Organizations: Near Optimizing Knowledge and Power Flows (Phase Two), Command and Control Research and Technology Symposium: The State of the Art and the State of the Practice, San Diego, CA, US, (2006).

[12] C. Bratianu and D. Andriessen, Knowledge as energy, 9th European Conference on Knowledge Management, Southampton Solent University, Southampton, Academic Publishing Limited, Reading UK (2008).

[13] C. Bratianu, Knowledgedynamics and Thermodynamics, Revista Informatica Economică, 12, 43 (2008).

[14] D. Andriessen, Knowledge as love. How metaphors direct our efforts to manage knowledge in organizations, 8th European Conference on Knowledge Management, Barcelona, Spain (2007)

[15] T.H. Davenport and L. Prusak, Working Knowledge: How Organizations Manage What They Know, Boston, MA, Harvard Business School Press, (1998).

[16] M.M. Appleyard, How Does Knowledge Flow? Interfirm Patterns in the Semiconductor Industry, in: C.W. Choo and N. Bontis (Eds.) The Strategic Management of Intellectual Capital and Organizational Knowledge, New York, NY, Oxford University Press, (2002).

[17] C.U. Ciborra and R. Andreu, Knowledge across Boundaries: Managing Knowledge in Distributed Organizations in: C.W. Choo, and N. Bontis (Eds.) The Strategic Management of Intellectual Capital and Organizational Knowledge, New York, NY, Oxford University Press, (2002).

[18] M.H. Boisot, Information Space: A Framework for Learning in Organizations, Institutions and Culture, London, Routledge (1995).

[19] M.H. Boisot, Knowledge Assets: Securing Competitive Advantage in the Information Economy, New York: Oxford University Press, (1998). 
[20] M. McLuhan, The Gutenberg Galaxy, Univ. Toronto Press (1962).

[21] E. P. Wigner, Proceedings of the American Philosophical Society, 94, No.5 October, (1950).

[22] M.J. Bates, Information and knowledge: an evolutionary framework for information science, Information Research 10, No. 4 (2005).

[23] I. Tuomi, Data Is More Than Knowledge: Implications of the Reversed Knowledge Hierarchy for Knowledge Management and Organizational Memory, Journal of Management Information Systems, 16103 (2000).

[24] J.J. Gibson, The Perception of the Visual World, Cambridge, MA, The Riverside Press, 1950.

[25] T.S. Kuhn, The Structure of Scientific Revolutions, Chicago, The University of Chicago Press, 1970.

[26] R. Dawkins, The Extended Phenotype: The Long Reach of the Gene, Oxford, UK, Oxford University Press, (1982).

[27] B. J. Loasby, The evolution of knowledge: beyond the biological model, Research Policy 31, 1227 (2002).

[28] The British Library Annual Report and Accounts 2004/2005

http://www.bl.uk/about/annual/2004to2005/pdf/statis tics.pdf.

[29] E. Schrödinger, What is Life? The Physical Aspect of the Living Cell. Based on Lectures delivered under the auspices of the Institute at Trinity College, Dublin, in February 1943, Cambridge: University Press. (1944).

[30] V. Aubuchon after US Department of Commerce, http://www.vaughns $\neg 1$-pagers.com/history/worldpopulation-growth.htm. (2004).

Prof. Tadeusz Wibig is a head of the Chair of Modeling the Teaching Processes at the Faculty of Physics and Applied Informatics of University of Łódź, Poland. He works also in the National center for Nuclear Studies in Cosmic Ray Laboratory. In research his interest is the cosmic ray physics and astrophysics, high energy particle physics, informatics and educational issues. He is a president of Lodz division of the Polish Physics Society.

Miss Punsiri Dam-o is PhD student at the University of Lodz, Poland. She finished Physics Education studies at Walailak University, Thailand. She is involved in the creation of remote controlled educational physics laboratory network. 